\title{
FITOTERÁPICOS: UMA POSSIBILIDADE NA CLÍNICA ODONTOPEDIÁTRICA
}

\author{
Bruna Balthazar SCHEFFELMEIER ${ }^{1}$ \\ bruna.scheffelmeier@yahoo.com.br \\ José Massao MIASATO² \\ jmassao@unigranrio.edu.br \\ Bárbara de Azevedo Abrahim VIEIRA ${ }^{3}$ \\ barbaraabrahim@pharma.ufrj.br
}

\section{RESUMO}

A fitoterapia ainda é um assunto pouco explorado na Odontologia. Uma grande vantagem dos fitoterápicos em relação aos produtos sintéticos é a menor toxicidade, fator de ainda maior peso quando se trata de odontopediatria. Com isso, o objetivo deste estudo foi avaliar, através de uma revisão da literatura, a eficácia dos produtos fitoterápicos como terapia alternativa de prevenção e tratamento de afecções orais comuns e controle da ansiedade em odontopediatria. Foi avaliada a eficácia dos fitoterápicos em situações como pulpotomia, controle de biofilme, tratamento de aftas e controle da ansiedade ao tratamento odontológico. Em todas as situações, os resultados foram positivos quanto ao uso da fitoterapia.

PALAVRAS-CHAVE: FITOTERAPIA; TERAPIAS COMPLEMENTARES; ODONTOPEDIATRIA; SAÚDE BUCAL

\section{PHYTOTHERAPICS: A POSSIBILITY IN THE PEDIATRIC DENTISTRY CLINIC}

\begin{abstract}
Phytotherapy is still a rarely explored subject in dentistry. A major advantage of phytotherapy compared to synthetic products is the lower toxicity, a factor of even greater weight when it comes to pediatric dentistry. Therefore, the objective of this study was to evaluate, through a literature review, the effectiveness of herbal products as an alternative therapy to prevention and treatment of common oral conditions in pediatric dentistry. The efficacy of phytotherapics was evaluated in situations such as pulpotomy, biofilm control, treatment of canker sores and anxiety control to dental treatment. In all situations, the results were positive regarding the use of herbal medicine.

KEY WORDS: PHYTOTHERAPY; COMPLEMENTARY THERAPIES; PEDIATRIC DENTISTRY; ORAL HEALTH

1 Aluna de graduação do Curso de Odontologia na Universidade do Grande Rio.

2 Mestre e Doutor em Odontopediatria. Professor Coordenador da disciplina de Odontopediatria na Universidade do Grande Rio.

3 Mestre em Ciências Aplicadas a Produtos para Saúde pela Universidade Federal do Rio de Janeiro, Doutora em Ciências Farmacêuticas pela Universidade Federal do Rio de Janeiro e Universidade de Coimbra (doutorado-sandwich), Pós Doutora em Modelagem Molecular. Professora adjunto da Faculdade de Farmácia da Universidade Federal do Rio de Janeiro.
\end{abstract}


ISSN 1983-5183

\section{INTRODUÇÃO}

A busca por conhecimento de terapias alternativas cresce cada vez mais na área da saúde. A fitoterapia tem-se mostrado uma eficiente alternativa na odontopediatria, geralmente com boa aceitação dos responsáveis pelos pacientes por ser uma opção de baixo custo e menor toxicidade, comparada aos fármacos sintéticos ${ }^{1}$.

Entende-se por tratamento fitoterápico a utilização dos medicamentos provenientes de plantas medicinais ou dos vegetais in natura para a cura ou prevenção de enfermidades e ferimentos ${ }^{2}$.

A terapia com plantas medicinais tem registros dos tempos primórdios da humanidade. No Brasil, as práticas são herdadas dos indígenas, as quais, mais tarde, se fundiram com o conhecimento dos africanos e colonizadores europeus, se tornando cultura popular². Esse tipo de terapia tem fortes valores históricoculturais, visto que a população, através da tradição oral ancestral, reconhece sua eficácia e legitimidade ${ }^{3}$.

No ano de 1978, a Organização Mundial da Saúde (OMS) demonstrou seu apoio às medicinas tradicionais ${ }^{2,4}$. Em 2006, o Ministério da Saúde aprovou a Política Nacional de Práticas Integrativas e Complementares no Sistema Único de Saúde, afirmando que a fitoterapia "incentiva o desenvolvimento comunitário, a solidariedade e a participação social"5. Além disso, a utilização de plantas medicinais é totalmente sustentável e viável no Brasil, pela sua vasta biodiversidade. Todos esses fatores justificaram a criação da Política Nacional de Plantas Medicinais e Fitoterápicos em junho do mesmo ano, que mais tarde serviu como base para o Programa Nacional de Plantas Medicinais e Fitoterápicos, implementado em $2009^{5,6}$.

Uma grande vantagem dos fitoterápicos em relação aos produtos sintéticos é a menor toxicidade, fator que tem maior peso na terapia de grupos especiais, como idosos, gestantes e crianças. A odontopediatria tem seu foco mais voltado para a prevenção do que para a cura. As principais manifestações orais como doença cárie e gengivite podem ser evitadas com a higiene propícia, porém os fitoterápicos podem ser grandes aliados, principalmente em áreas de pouco recurso financeiro?

O objetivo deste estudo foi buscar evidências da eficácia dos produtos fitoterápicos, visando apresentar alternativas de prevenção e tratamento de afecções bucais e controle da ansiedade ao tratamento odontológico infantil.

\section{MÉTODOS}

O método utilizado foi a busca de artigos publicados sobre o tema, utilizando as principais bases de dados LILACS, Pubmed e Scielo. Foram priorizadas as publicações a partir do ano 2000, com exceção da declaração de Alma-Ata (1978). A pesquisa foi feita com descritores nos idiomas Português e Inglês: "fitoterapia odontopediatria", "fitoterapia odontologia", "phytotherapy pediatric dentistry", "fitoterapia" e "phytotherapy".

\section{REVISÃO DA LITERATURA/DISCUSSÃO}

Quando bem conhecida e aplicada, a fitoterapia é uma grande aliada da odontopediatria, já que auxilia desde o condicionamento do paciente ao tratamento propriamente dito ${ }^{8}$. Trata-se de um tema vantajoso a ser explorado, visto que a prática já é utilizada pelos pacientes, principalmente em locais rurais 
ISSN 1983-5183

em diversas situações ${ }^{7}$. Além de ser uma alternativa menos tóxica comparada aos fármacos tradicionais, é também uma forma de valorizar a biodiversidade do país ${ }^{1,5}$.

Uso de produtos fitoterápicos na Odontopediatria

\section{Pulpotomia}

No estudo conduzido por Gupta et al. ${ }^{9}$ (2010), um gel de Aloe vera foi aplicado após a pulpotomia em quinze pacientes, com o objetivo de evitar inflamações ou infecções pós-tratamento. Todos obtiveram resultados satisfatórios, sem presença de abscesso, dor ou mobilidade. Os pacientes foram monitorados dois meses após o procedimento e foram comprovados os resultados positivos. Os dentes decíduos foram, então, extraídos e avaliados histologicamente, e foi constatado que os tecidos estavam todos saudáveis.

A efetividade do Aloe vera também foi testada em tecidos pulpares de ratos, e obteve bons resultados: concluiu-se que o produto é biocompatível e estimula a formação de dentina reparadora. Seu efeito terapêutico foi similar ao do hidróxido de cálcio ${ }^{10}$.

\section{Controle de biofilme}

A doença cárie é o achado de maior prevalência na odontopediatria. Trata-se de um problema multifatorial e os fatores etiológicos são: dieta, higiene oral para controle mecânico e químico do biofilme, levando em conta qualidade e quantidade de escovações, nível socioeconômico e nível de educação dos pais da criança11.

Além da cárie, a deficiência no controle do biofilme causa também a gengivite, que é muito comum em crianças por ainda não apresentarem maturidade para compreender a importância da saúde oral' ${ }^{12,13}$.

É importante o profissional avaliar a realidade socioeconômica do paciente, pois é um fator de risco para doenças periodontais. $O$ diagnóstico precoce da doença é primordial para evitar um futuro quadro de periodontite ${ }^{12}$.

Clinicamente, há sangramento à sondagem, coloração avermelhada e edema. 0 tratamento é feito com o controle de placa através da higienização adequada, podendo ou não ser auxiliada por colutórios, com acompanhamento profissionall ${ }^{13}$.

Os dentifrícios herbais têm demonstrado resultados muito positivos no controle do biofilme, prevenindo cárie e gengivite. Em um estudo foi avaliada a ação de dentifrícios e colutórios à base de extrato hidroalcoólico de Lippia sidoides Cham (alecrim pimenta), e o resultado mostrou a eficiência dos produtos fitoterápicos na redução do índice de biofilme dentário comparados ao dentifrício comum ${ }^{14}$.

Outro estudo conduzido por Albuquerque et al. ${ }^{15}$ (2008) confirma a atividade antimicrobiana do extrato de Lippia sidoides Cham in vitro sobre os microrganismos Streptococcus mutans, Streptococcus sanguis, Streptococcus mitis, Streptococcus sobrinus e Lactobacillos casei em comparação à clorexidina 0,12\%.

Uma avaliação clínica comparando dentifrício comum e à base de extrato de Rosmarinus officinalis Linn (alecrim) não demonstrou diferença significativa entre os dois produtos: ambos obtiveram a mesma eficácia clínica ${ }^{16}$.

Em duas análises clínicas do efeito de bochechos fitoterápicos à base de extrato de romã e de 
ISSN 1983-5183

camomila, concluiu-se que são eficazes na redução do biofilme dental e sangramento gengival. Ambos os produtos a presentam ações antimicrobianas e anti-inflamatórias e se mostraram aptos para a recuperação e manutenção da saúde periodontal, embora o extrato de romã tenha se mostrado menos eficaz na redução do biofilme dental em casos de gengivite crônica e periodontite crônica, comparado ao extrato de camomila e à clorexidina ${ }^{17,18}$.

Aftas

Uma das patologias mais comuns da mucosa oral, a úlcera aftosa pode ter variadas etiologias: traumas, estresse, predisposição genética, alergias, deficiências nutricionais, agentes infecciosos, anormalidades hematológicas, entre outros. Variam conforme tamanho, forma e número de lesões ${ }^{13}$.

O tratamento fitoterápico para esse tipo de afecção bucal é feito com plantas de ação anti-inflamatória, como o cravo-da-índia (Syzygium aromaticun L.), tanchagem (Plantago major L.), sálvia (Salvia officinalis L.), romã (Punica granatum), malva (Malva sylvestris), entre outras ${ }^{8,19}$.

Uma revisão de literatura realizada por Martín Mauriño e Martínez-Sahuquillo20 (2016) reuniu artigos de caso-controle investigando a eficácia de plantas como Murta-comum (Myrtus communis), babosa (Aloe vera), mirra (Commiphora myrrha), berberina (composto extraído de plantas) e alicina (substância presente no alho). Os efeitos foram comparados a um placebo e ao corticosteroide sintético triancinolona acetonida e foi constatada a eficácia dos fitoterápicos: reduziram a dor, o tamanho da lesão e houve aceleração na cicatrização sem efeitos adversos. Apesar de não terem superado os resultados do tratamento com corticosteroide, concluiu-se que a terapia natural pode ser usada em pacientes que não querem fazer uso do medicamento sintético por longo período de tempo.

\section{Controle da ansiedade ao tratamento odontológico}

Um grande desafio na área da odontopediatria é lidar com a ansiedade do paciente ao tratamento. A origem desse problema pode ser de experiências anteriores negativas, influência dos pais, ou mesmo pelo medo do desconhecido. $\mathrm{O}$ condicionamento do paciente odontofóbico deve ser feito, primeiramente, com as técnicas tradicionais de controle comportamental, como a adequação do ambiente do consultório, eliminando objetos e sons que possam assustar, utilização de aromas de óleos essenciais na sala de espera, falar-mostrar-fazer, controle do tom de voz, reforço positivo, distração, entre outros ${ }^{21}$.

Quando as técnicas de condicionamento não são suficientes para o controle da fobia da criança, é possível lançar mão de medicamentosfitoterápicos para evitar o uso de sedativos, como os benzodiazepínicos. Um estudo conduzido por Dantas ${ }^{22}$ (2014) comparou os efeitos da Passiflora incarnata e do midazolam no controle da ansiedade em pacientes submetidos à exodontia de molares inclusos. O fitoterápico obteve excelentes resultados, comprovando seu efeito ansiolítico similar ao medicamento sintético, mantendo estáveis os níveis de pressão arterial e frequência cardíaca, o que aponta sua segurança e eficácia. A Passiflora incarnata pode ser utilizada em crianças a partir de dois anos de idade, e as únicas contraindicações para o uso desse medicamento são hipersensibilidade e intolerância à lactose ${ }^{8}$.

Um estudo realizado comparando a efetividade da Valeriana officinalis $L$. e um placebo no controle da ansiedade em pacientes submetidos a exodontias bilaterais de terceiros molares inferiores inclusos apontou que o fitoterápico possui efeitos ansiolíticos e pode ser utilizado na sedação consciente ${ }^{23}$. A Valeriana não possui contraindicações para o uso pediátrico ${ }^{8}$. 
ISSN 1983-5183

\section{CONCLUSÃO}

Com base na literatura consultada, é lícito concluir que a fitoterapia é uma alternativa eficaz na prevenção e tratamento de afecções orais comuns em Odontopediatria.

\section{REFERÊNCIAS BIBLIOGRÁFICAS}

1. CARVALHO IO. Atividade antibacteriana de óleos essenciais e cremes dentais fitoterápicos em bactérias cariogênicas [Dissertação]. Minas Gerais: Universidade Federal de Viçosa - UFV; 2016.

2. ALVES AR, Silva MJP. O uso da fitoterapia no cuidado de crianças com até cinco anos em área central e periférica da cidade de São Paulo. Rev EsC Enferm USP 2003 dez.;37(4):8591.

3. SANTOS RL, Guimaraes GP, Nobre MSC, Portela AS. Análise sobre a fitoterapia como prática integrativa no Sistema Único de Saúde. Rev bras plantas med 2011 13(4):486-91.

4. ORGANIZAÇÃO Mundial De Saúde (OMS). Alma-Ata. 1978: cuidados primários de saúde: relatório da Conferência Internacional sobre Cuidados Primários de Saúde Alma-Ata, URSS, 6-12 de setembro de 1978. Brasil: UNICEF; 1979 [Acesso em: 26 março 2018]; Disponível em: http://apps.who.int/iris/bitstream/handle/10665/39228/9241800011 por. pdf:jsessionid=23A0CCB7D7EDB62EC24F27109C77A51D? sequence=5.

5. BRASIL. Ministério Da Saúde. Secretaria De Ciência. Tecnologia E Insumos Estratégicos. Política nacional de plantas medicinais e fitoterápicos. Brasília: Ministério da Saúde; 2006 [Acesso em: 26 março 2018]; Disponível em: http://bvsms.saude.gov.br/bvs/publicacoes/politica nacional fitoterapicos.pdf.

6. BRASIL. Ministério Da Saúde. Secretaria De Ciência. Tecnologia E Insumos Estratégicos. Programa nacional de plantas medicinais e fitoterápicos Brasília: Ministério da Saúde; 2009 [Acesso em: 27 março 2018]; Disponível em: http://bvsms.saude.gov.br/bvs/publicacoes/programa nacional plantas medicinais fitoterapicos.pdf.

7. CARVALHO GD, Souza ED, Souza LA, Porfirio LC, Siqueira HM. Perfil de famílias interioranas que fazem uso de plantas medicinais PUBVET [Periódico on-line].2008; 2(12). [Acesso em: 27 março 2018] Disponível em: http://pubvet.com.br/material/Carvalho185.pdf.

8. MONTEIRO MHDA. Fitoterapia na odontologia: levantamento dos principais produtos de origem vegetal para saúde bucal [Tese]. Rio de Janeiro: Fundação Oswaldo Cruz FIOCRUZ; 2014. $219 f$.

9. GUPTA N, Bhat M, Devi P, Girish. Aloe-vera: a nature's gift to children. Int J Clin Pediatr Dent 2010 May-Aug;3(2):87-92.

10. GALA-GARCíA A, Teixeira KIR, Mendes LL, Sobrinho APR, Santos VR, Cortes ME. Effect of aloe vera on rat pulp tissue. Pharmaceutical Biology 2008 46(5):302-8.

11. LOSSO EM, Tavares MCR, Silva JYB, Urban CA. Cárie precoce e severa na infância: uma abordagem integral. J Pediatr 2009 ago;85(4):295-300. 
ISSN 1983-5183

12. CHAVES RA, Neves AM, Miranda KCO, Passos IA, Oliveira AFB. Consultório odontológico na escola: análise da saúde gengival e do nível de higiene oral. $R G O$ - Rev Gaúcha Odontol, Porto Alegre 2011 jan/mar;59(1):29-34.

13. NEVILLE BW, Damm DD, Allen CM, Bouquot JE. Patologia: oral \& maxilofacial. 2. ed. Rio de Janeiro: Guanabara Koogan; 2004.

14. NUNES RS, Lira AA, Lacerda CM, Silva DOB, Silva JA, Santana DP. Obtenção e avaliação clínica de dentifrícios à base do extrato hidroalcoólico da Lippia sidoides Cham (Verbenaceae) sobre o biofilme dentário. Rev Odontol UNESP 2006 out-dez ;35(4):275-83.

15. ALBUQUERQUE ACL, Pereira MSV, Pereira JV, Costa MRM, Higino JS. Efeito antimicrobiano do extrato da Lippia sidoides Cham. sobre microrganismos cariogênicos. Arq Odontol 2008 44(4):5-10.

16. TENÓRIO LA. A avaliação clínica da atividade do dentifrício à base do extrato de Rosmarinus officinalis Linn. (alecrim) sobre o biofilme dental: um estudo preliminar [Dissertação]. Recife: Universidade Federal de Pernambuco - UFP; 2014.

17. BARBOSA DN. Análise clínica do efeito de fitoterápicos na redução do biofilme dental e sangramento gengival na doença periodontal [Dissertação]. Campina Grande: Universidade Estadual da Paraíba - UEPB; 2011.

18. BELÉM NM. Análise clínica e microbiológica do efeito de fitoterápicos na redução do biofilme dental e do sangramento gengival na doença periodontal [Dissertação]. Campina Grande: Universidade Estadual da Paraíba - UEPB; 2011.

19. OLIVEIRA FQ, Gobira B, Guimarães C, Batista J, Barreto M, Souza M. Espécies vegetais indicadas na odontologia. Rev bras farmacogn 2007 set;17(3):466-76.

20. MARTÍN Mauriño AM, Martínez-Sahuquillo Márquez Á. Tratamiento de la estomatitis aftosa recidivante [Monografia]. Sevilla: Universidad de Sevilla; 2016.

21. SCHNEIDER JO. Abordagem da ansiedade em Odontopediatria: revisão de literatura [Monografia]. Curitiba: Universidade Federal do Paraná - UFPR; 2013.

22. DANTAS LP. Efeitos da passiflora incarnata e do midazolam no controle da ansiedade em pacientes submetidos à exodontia de tercerios molares inclusos [Dissertação]. Aracaju: Universidade Federal de Sergipe - UFS; 2014.

23. PINHEIRO MLP. Avaliação dos efeitos da Valeriana officinalis $L$ no controle da ansiedade em pacientes submetidos a exodontias de terceiros molares mandibulares inclusos [Dissertação]. Piracicaba: Universidade Estadual de Campinas - UEC; 2003.

RECEBIDO EM 23/10/2017

ACEITO EM 23/10/2017 